\title{
Transarterial chemoembolization combined with sorafenib for the treatment of hepatocellular carcinoma with hepatic vein tumor thrombus
}

\author{
This article was published in the following Dove Press journal: \\ OncoTargets and Therapy \\ 12 July 2016 \\ Number of times this article has been viewed
}

\author{
Yong-Fa Zhang ${ }^{1-3, *}$ \\ Wei Wei ${ }^{1-3, *}$ \\ Jia-Hong Wang ${ }^{1-3, *}$ \\ Li Xu ${ }^{1-3}$ \\ Pei-En Jian ${ }^{1-3}$ \\ Cheng-Zuo Xiao ${ }^{4}$ \\ Xiao-Ping Zhong ${ }^{1-3}$ \\ Ming Shi ${ }^{1-3}$ \\ Rong-Ping Guo ${ }^{1-3}$ \\ 'Department of Hepatobiliary \\ Oncology, Sun Yat-sen University \\ Cancer Center, ${ }^{2}$ State Key Laboratory \\ of Oncology in South China, \\ ${ }^{3}$ Collaborative Innovation Center \\ for Cancer Medicine, Guangzhou, \\ ${ }^{4}$ Department of General Surgery, \\ Shenzhen Shajing Affiliated Hospital \\ of Guangzhou Medical University, \\ Shenzhen, People's Republic of China \\ *These authors contributed equally \\ to this work
}

\begin{abstract}
Objective: To compare the treatment outcomes of sorafenib plus transarterial chemoembolization (TACE) vs TACE alone in patients with hepatocellular carcinoma (HCC) and hepatic vein tumor thrombus (HVTT).

Methods: Twenty patients who were initially diagnosed with HCC and HVTT and received TACE combined with sorafenib during February 2009 to October 2013 were included in the study. To minimize selection bias, these patients were compared with 60 case-matched controls selected from a pool of 81 patients (in a 1:3 ratio) who received TACE alone during the same period. The primary end point was overall survival (OS). The secondary end points were time to progression, disease control rate, and adverse events.

Results: After a median follow-up period of 12.5 months (range, 1.03-44.23 months), the OS of the combined group was found to be significantly higher compared with the monotherapy group (14.9 vs 6.1 months, $P=0.010$ ). The time to progression was found to be significantly longer in the combined group ( 4.9 vs 2.4 months, $P=0.016$ ). Univariate and multivariate analyses revealed that the treatment allocation was an independent predictor of OS.

Conclusion: Sorafenib plus TACE was well tolerated and was more effective in treating patients with advanced HCC and HVTT. Future trials with prospective larger samples are required to validate these results.
\end{abstract}

Keywords: hepatocellular carcinoma, hepatic vein tumor thrombus, prognosis, adverse events

\section{Introduction}

Hepatocellular carcinoma (HCC) is the fifth most frequently diagnosed cancer worldwide and the second most frequent cause of cancer death. ${ }^{1}$ Despite recent improvements in surveillance programs, a high percentage of patients with HCC are not diagnosed until the disease progresses to an advanced stage (Barcelona Clinic Liver Cancer stage C), for which there is no curative therapy, resulting in a dismal prognosis. ${ }^{2}$ Sorafenib, an inhibitor of cancer cell proliferation and tumor angiogenesis, has been approved for treatment of advanced HCC based on two randomized, placebocontrolled trials. ${ }^{3,4}$ However, in those trials, the prognosis among the patients with $\mathrm{HCC}$ remained modest, and the local tumor response rate was low.

Transarterial chemoembolization (TACE) is the standard treatment for intermediate-stage HCC. ${ }^{2,5}$ Recently, some studies have proved that TACE can serve as a safe procedure in selected HCC patients with portal vein tumor thrombus (PVTT) or hepatic vein tumor thrombus (HVTT). ${ }^{6-10}$ However, the hypoxia caused by TACE in surviving HCC tumor cells leads to the release of angiogenic growth factors, which
Correspondence: Rong-Ping Guo Ming Shi

Department of Hepatobiliary Oncology, Sun Yat-sen University Cancer Center,

65I Dongfeng Road East, Guangzhou

$\mathrm{Tel} / \mathrm{fax}+86208734$ 2266;

+862087343938

Email guorongp@mail.sysu.edu.cn; shiming@sysu.edu.cn 
results in tumor recurrence or metastases and a worse prognosis. ${ }^{11-13}$ Moreover, an increase in vascular endothelial growth factor (VEGF) levels has been reported in patients treated with TACE and to predict poor prognosis. ${ }^{12,14}$ The use of sorafenib can significantly counteract the tyrosine kinase receptors VEGFR-2/3 and platelet-derived growth factor receptor (PDGFR)-b, which decreases the post-TACE VEGF and PDGF upregulation and further enhances the efficacy of TACE. ${ }^{15,16}$ Besides, sorafenib combined with cytotoxic drug shows a synergism that sorafenib inhibits the Ras/Raf/MEK/ERK pathway, which will prevent activation of the multidrug resistance pathway that leads to the failure of chemotherapy in treating HCC. ${ }^{17}$ The combined regimen of sorafenib with cisplatin has been tested in clinical trials in patients with pediatric $\mathrm{HCC},{ }^{18}$ gastric cancer, ${ }^{19}$ lung cancer, ${ }^{20}$ and nasopharyngeal carcinoma, ${ }^{21}$ with favorable outcomes reported. Several studies have also tested TACE combined with sorafenib and indicated its safety and efficacy in the treatment of HCC with PVTT. ${ }^{15,22}$ Although the involvement of HVTT in HCC has been less frequently observed $(2.3 \%-26.2 \%)$ compared with that of the PVTT, ${ }^{23-25}$ little is known about the clinical outcomes of sorafenib combined with TACE for HVTT. ${ }^{26}$

So, we conducted this study to compare the safety and efficacy of sorafenib combined with TACE in patients with advanced HCC and HVTT compared with TACE monotherapy.

\section{Materials and methods Study design}

This study complied with the Health Insurance Portability and Accountability Act regulations and was approved by the Ethics Committee of the Sun Yat-sen University Cancer Center. From February 2009 to October 2013, a retrospective review was carried out of all the patients with HCC and HVTT who were admitted to the Sun Yat-sen University Cancer Center. Diagnosis of HCC was based on the European Association for the Study of the Liver/American Association for the Study of Liver Diseases guidelines. ${ }^{2}$ The presence of tumor thrombus was identified by Doppler ultrasound, enhanced computed tomography (CT) scan, or magnetic resonance imaging. A total of 124 patients diagnosed with HCC and HVTT were detected from the database. Eventually, 13 patients who had hepatic resection or other non-TACE intervention were excluded, and 101 patients who received either TACE alone or TACE combined with sorafenib as the initial treatment for HCC and HVTT were included. Of these, 20 patients who received TACE combined with sorafenib as the initial treatment for HCC and HVTT (the TACE-sorafenib group) were matched in a 1:3 ratio with those selected from a pool of 81 patients who were treated with TACE alone as an initial treatment (the TACE group). The patients in the two groups were matched as closely as possible based on the following factors: 1) extrahepatic metastasis, 2) vascular invasion, 3) tumor burden, 4) liver function, 5) Eastern Cooperative Oncology Group performance status (ECOG PS) and age, 6) etiology, and 7) alpha-fetoprotein level. Survival outcomes of the TACE group were not known at the time of matching. Demographic and clinical data, response to treatment, and survival status of patients were analyzed retrospectively.

\section{Inclusion and exclusion criteria}

The inclusion criteria for the study population were as follows: (a) age between 18 and 75 years, (b) diagnosis of HCC without antitumor therapy, (c) presence of HVTT on imaging, which was categorized based on the Japanese staging system, ${ }^{27}$ (d) an ECOG PS of 0-1, (e) Child-Pugh class A liver disease, and (f) one or more measurable lesions in the liver. Patients who were medically unsuitable for TACE (eg, with extensive intrahepatic disease and cirrhosis), had gastrointestinal bleeding, or had received other treatments (radiofrequency ablation, percutaneous ethanol injection, or iodine-125 seed implantation) besides TACE during this study were excluded. Other exclusion criteria included hepatic encephalopathy, history of cardiac disease, uncontrolled hypertension ( $\geq 150 / 100 \mathrm{mmHg}$ ), HIV infection, a second primary malignancy, and known contraindications to sorafenib.

However, there is no concrete evidence establishing TACE-sorafenib as an optimal treatment strategy for HCC with HVTT. The patients were fully informed in detail about the possible benefits and risks of treatment with TACE or TACE-sorafenib, and they finally decided the choice of treatment. Before the patients underwent initial TACE treatment and were diagnosed with disease progression by imaging after TACE treatment, the TACE-sorafenib treatment strategy was recommended once again by our physician. If the patients agreed to the physician's recommendation, sorafenib was administered as soon as possible. Those patients who refused sorafenib received TACE only. Written consent was obtained from each patient before treatment.

\section{TACE procedure}

TACE was carried out by using techniques we described previously. ${ }^{7}$ Before the TACE procedure, angiography of 
the superior mesenteric and hepatic artery was performed to assess the portal vein patency, vascular anatomy, and tumor vascularity. Depending on the arterial supply of the tumor identified by arteriography, a 2.7 Fr microcatheter (Terumo, Tokyo, Japan) was superselectively placed into the feeding arteries of the tumor and the tumor thrombus for selective embolization, which was carried out with an embolization suspension consisting of mitomycin $\mathrm{C}$ (6 mg), lobaplatin $(50 \mathrm{mg})$, epirubicin $(50 \mathrm{mg})$, iodized oil (Lipiodol; Guerbet, Villepinte, France), and gelatinsponge particles.

\section{Sorafenib administration}

Sorafenib treatment was initiated at a dose of $400 \mathrm{mg}$ twice daily. The combined group was given sorafenib on an interrupted schedule, with an interval of 4-7 days before and after any TACE session. Sorafenib dose reduction was based on the presence of toxicity. Patients were encouraged to insist on continuing the sorafenib treatment if the toxicity was manageable. After observation of progressive disease (PD), the choice of continuing sorafenib treatment was first discussed between the patient and the physician, but the final decision was made by the patient. If the treatment was continued, informed consent was obtained, and the therapy of sorafenib was maintained until a deterioration in the ChildPugh score to $\mathrm{C}$ or the ECOG PS score to 4 was found, or until the appearance of intolerable complications or death. ${ }^{13}$

\section{Assessments}

The primary end point of the study was overall survival (OS). The secondary end points included time to progression (TTP), disease control rate (DCR: complete response $[\mathrm{CR}]+$ partial response $[\mathrm{PR}]+$ stable disease $[\mathrm{SD}]$ ) according to the modified Response Evaluation Criteria in Solid Tumors, ${ }^{28}$ and treatment-associated adverse events (AEs). OS was defined as the time from the first TACE procedure until death or the last follow-up in censored patients. TTP was defined as the time from the start of the first TACE procedure until the date the tumor progression was confirmed radiologically. Among patients who underwent treatment with sorafenib after disease progression, TTP was revalued only for those who showed disease control on imaging. AEs were graded using the Common Terminology Criteria for AEs (version 3.0) from the National Cancer Institute. ${ }^{29}$ In the two groups, AEs that occurred within 4 weeks after TACE treatment were recorded. In the TACE-sorafenib group, sorafenib-related AEs were monitored until discontinuation of sorafenib.

\section{Follow-up}

Each follow-up session included a detailed history and physical examination, laboratory tests, and abdominal contrast material-enhanced three-phase dynamic spiral CT or magnetic resonance imaging. Patients visited the clinic every 4-6 weeks and at the end of treatment for assessment of compliance and safety, and determination of side effects. TACE was repeated on demand if residual viable tumor tissue was evident at sequential dynamic liver CT without deterioration of hepatic function. This study was censored on November 30, 2014.

\section{Statistical analysis}

Continuous variables were summarized as the mean and range. Categorical variables were expressed as frequencies and percentages. Survival was assessed according to the Kaplan-Meier method. Differences in survival between the two groups were compared using log-rank test, and predictors of OS were determined by Cox regression analysis. DCR was compared between the two treatment groups using Student's $t$-test. All statistical tests were two sided, and a significant difference was considered when $P$-value was $<0.05$.

\section{Results \\ Patient characteristics}

From February 2009 to October 2013, a total of 80 patients with advanced HCC and HVTT were eventually enrolled. Of these, 20 patients were allocated to the TACE-sorafenib group. Sorafenib treatment was initiated immediately for eleven patients $(55 \%)$ after the first-session TACE, and nine patients $(45 \%)$ were administered sorafenib after being diagnosed with disease progression. The overall interval between TACE and sorafenib treatments was 4.0 months (range, 0.1-28.3 months). The characteristics of patients such as age, sex, ECOG PS, liver function, and tumor burden, as well as the presence of vascular invasion and/or metastasis, are shown in Table 1.

Patients in the TACE-sorafenib group and patients in the TACE group underwent repeated TACE, with a mean of 2.0 (range, 1-4) and 1.30 (range, 1-3) TACE procedures per patient, respectively. Five patients were subjected to dose reductions and then reverted to a regular dose after relief from AEs. Two patients were subjected to drug interruption for a period of 15 and 26 days due to the AEs and then restored to a regular dose after relief from AEs. A median of $755.7 \mathrm{mg}$ (range, 500-800 mg) of sorafenib was administered daily over a median of 9.0 months $(95 \%$ confidence interval [CI]: 3.7-14.3 months) in the TACE-sorafenib group. 
Table I Pretreatment baseline characteristics of patients

\begin{tabular}{|c|c|c|c|}
\hline Characteristics & TACE-sorafenib group $(n=20)$ & TACE group $(n=60)$ & $P$-value \\
\hline \multicolumn{4}{|l|}{ Epidemiology } \\
\hline Age, years & $46.6 \pm 11.0$ & $48.6 \pm 7.1$ & 0.447 \\
\hline Male/female, n (\%) & $18 / 2(90 / 10)$ & $58 / 2(97 / 3)$ & 0.259 \\
\hline Etiology, n (\%) & & & 0.454 \\
\hline Hepatitis B carrier & $16(80)$ & $53(88)$ & \\
\hline Others & $4(20)$ & $7(12)$ & \\
\hline ECOG performance $(0 / 1)$ & $17 / 3(85 / 15)$ & $52 / 8(87 / 13)$ & 1.000 \\
\hline \multicolumn{4}{|l|}{ Liver function } \\
\hline Liver cirrhosis (yes/no), n (\%) & $18 / 2(90 / 10)$ & $52 / 8(87 / 13)$ & 1.000 \\
\hline Platelet count $\left(10^{9} / \mathrm{L}\right)$ & $234.1 \pm 89.1$ & $214.5 \pm 73.8$ & 0.331 \\
\hline Prothrombin time (seconds) & $12.2 \pm 1.5$ & $\mid 2.7 \pm 1.1$ & 0.138 \\
\hline Alanine aminotransferase $(U / L)$ & $75.3 \pm 44.7$ & $85.0 \pm 51.4$ & 0.457 \\
\hline Serum albumin $(g / L)$ & $40.3 \pm 4.6$ & $39.1 \pm 4.1$ & 0.273 \\
\hline Total bilirubin (mmol/L) & $17.6 \pm 6.6$ & $16.0 \pm 6.1$ & 0.319 \\
\hline \multicolumn{4}{|l|}{ Tumor burden } \\
\hline Tumor size $(\mathrm{cm})$ & $9.6 \pm 4.0$ & $10.3 \pm 3.4$ & 0.421 \\
\hline Tumor number $(\leq \mathrm{I} />\mathrm{I}), \mathrm{n}(\%)$ & $6 / 14(30 / 70)$ & $22 / 38(37 / 63)$ & 0.588 \\
\hline Tumor extent (unilobar/bilobar), n (\%) & $14 / 6(70 / 30)$ & $44 / 16(73 / 27)$ & 0.772 \\
\hline Alpha-fetoprotein level ( $\leq 400 />400)(n g / m L), n(\%)$ & $7 / 13(35 / 65)$ & $18 / 42(30 / 70)$ & 0.676 \\
\hline \multicolumn{4}{|l|}{ Vascular invasion } \\
\hline Combined PVTT (yes/no), n (\%) & $8 / 12(40 / 60)$ & $29 / 31(48 / 52)$ & 0.517 \\
\hline Location of HVTT (Vv2/Vv3), n (\%) & $7 / 13(35 / 65)$ & $21 / 39(35 / 65)$ & 1.000 \\
\hline \multicolumn{4}{|l|}{ Extrahepatic metastasis } \\
\hline Lung/lymph nodes/bones/suprarenal gland, n (\%) & $\mathrm{I} / \mathrm{I} / \mathrm{I} / \mathrm{I}(25 / 25 / 25 / 25)$ & $3 / 3 / 3 / 3(25 / 25 / 25 / 25)$ & 1.000 \\
\hline
\end{tabular}

\section{Safety}

AEs related to TACE within 4 weeks after treatment are shown in Table 2. No significant difference between the two groups was found for grades 3-5 TACE-related AEs. The most common AEs related to sorafenib treatment were hand-foot skin reaction (HFSR; 70.0\%), diarrhea (45.0\%), and fatigue (35\%), most of which were grade 1 or 2 . Seven drug-related AEs of grades 3-5 were reported (HFSR, $n=2$; diarrhea, $n=2$; bleeding, $n=1$; hypertension, $n=1$; rash, $n=1$ ). There were no treatment-related deaths in this trial. All drugrelated AEs are listed in Table 3.

\section{Efficacy analysis}

After a median follow-up period of 12.5 months (range, 1.0344.23 months), 65 patients died, and 15 patients survived. The median survival time of the TACE-sorafenib group was 14.9 (95\% CI: 6.8-23.0) months and that of TACEalone group was $6.1(95 \% \mathrm{CI}$ : $4.0-8.1)$ months $(P=0.010)$ (Figure 1). The rates of radiologically confirmed CR, PR, SD, and PD after TACE and during sorafenib treatment are shown in Table 4. In the TACE-sorafenib group, no patients had $\mathrm{CR}$, ten patients $(50 \%)$ showed $\mathrm{PR}$, and six patients $(30 \%)$ showed SD. PD occurred in four patients (20\%). The DCR
$(\mathrm{CR}+\mathrm{PR}+\mathrm{SD})$ based on the modified Response Evaluation Criteria in Solid Tumors assessment was $80.0 \%$ among the patients treated with TACE and sorafenib, compared with $43.3 \%$ in patients treated with TACE alone $(P=0.004)$. The median TTP was 4.9 months (95\% CI: 3.7-6.0 months) for the combination therapy group and 2.4 months $(95 \% \mathrm{CI}$ : 1.3-3.4 months) for the TACE-alone group. Combination treatment significantly improved TTP compared with TACE alone $(P=0.016)$ (Figure 2).

\section{Univariate and multivariate analysis of predictors of OS}

The predictors of the OS in univariate and multivariate analyses are shown in Table 5. Alanine transferase, combined PVTT, and treatment allocation were found to be the significant predictors of OS in the univariate analysis. In multivariate regression analysis, treatment allocation (hazard ratio $=2.16 ; 95 \%$ CI: $1.18-3.94 ; P=0.012$ ) was found to be a significant predictor of OS.

\section{Discussion}

The efficacy of sorafenib in advanced HCC has been demonstrated and brought great hope. ${ }^{30}$ Recently, the use of 
Table 2 Adverse events related to TACE in patients with HVTT in the two groups

\begin{tabular}{|c|c|c|c|c|c|}
\hline \multirow[t]{2}{*}{ Incidence of complications } & \multicolumn{2}{|c|}{ TACE-sorafenib group $(n=20)$} & \multicolumn{2}{|c|}{ TACE group $(n=60)$} & \multirow{2}{*}{$\begin{array}{l}\text { P-value } \\
\text { (grades 3-5) }\end{array}$} \\
\hline & Any grade & Grades 3-5 & Any grade & Grades 3-5 & \\
\hline Postembolization syndrome & & & & & 0.358 \\
\hline Fever & 15 & I & 41 & I & \\
\hline Pain & 9 & 0 & 36 & 0 & \\
\hline Vomiting & 7 & 2 & 13 & 3 & \\
\hline Blood & & & & & 1.000 \\
\hline Platelets & 3 & I & 17 & 4 & \\
\hline Hemoglobin & 2 & I & 7 & 4 & \\
\hline Leukocytes & I & I & I & 0 & \\
\hline Liver function change & & & & & 0.102 \\
\hline Ascites/pleura effusion & 3 & 4 & 12 & 3 & \\
\hline Edema:limb & 2 & 0 & 3 & 0 & \\
\hline Liver dysfunction & 9 & 0 & 24 & I & \\
\hline Gastrointestinal bleeding & 1 & 0 & 1 & 0 & 0.440 \\
\hline Renal failure & I & 0 & 0 & 0 & 0.250 \\
\hline
\end{tabular}

Abbreviations: TACE, transarterial chemoembolization; HVTT, hepatic vein tumor thrombus.

sorafenib in combination with TACE for patients with HCC and PVTT was recommended. ${ }^{22,31}$ Our study demonstrated that, compared with TACE alone, sorafenib combined with TACE significantly improved the outcome in patients with advanced HCC and HVTT. Importantly, there were no unexpected side effects, and combination therapy did not appear to lead to worse AEs than observed with TACE alone. Moreover, multivariate analysis revealed that treatment allocation was a significant predictor of prognosis.

It has been postulated that the high recurrence rate after TACE results from proangiogenic factors that are triggered by hypoxic tumor changes caused by TACE. This increase in hypoxia directly upregulates HIF-1a, which, in turn, increases expression of various proangiogenic proteins and receptors (including VEGF and PDGFR). ${ }^{32,33}$ However, a decrease in plasma VEGF levels was noted in patients treated with TACE and sorafenib. ${ }^{16}$ The combined treatment with TACE plus an antiangiogenic agent may provide complementary inhibition of neovascularization and tumor growth. ${ }^{30,34}$ Thus,

Table 3 Adverse events related to sorafenib administration in the TACE-sorafenib group

\begin{tabular}{lll}
\hline Adverse events & \multicolumn{2}{l}{ TACE-sorafenib group $(\mathbf{n}=\mathbf{2 0})$} \\
\cline { 2 - 3 } & Any grade & Grades 3-5 \\
\hline Hand-foot skin reaction & $12(60)$ & $2(10)$ \\
Diarrhea & $7(35)$ & $2(10)$ \\
Fatigue & $7(35)$ & 0 \\
Alopecia & $6(30)$ & 0 \\
Bleeding & $5(25)$ & $\mathrm{I}(5)$ \\
Hypertension & $4(20)$ & $\mathrm{I}(5)$ \\
Rash & $4(20)$ & $\mathrm{I}(5)$ \\
Weight loss & $3(15)$ & 0 \\
Voice changes & $2(10)$ & 0
\end{tabular}

Note: Data presented as n (\%).

Abbreviation: TACE, transarterial chemoembolization. early administration of sorafenib might be able to suppress the effect of early surge of VEGF and possibly other angiogenic factors. Three models for the combination of sorafenib with TACE have been reported by Strebel and Dufour: the sequential approach, the interrupted approach, and the continuous approach. ${ }^{35}$ Several studies have showed that TACE in combination with sorafenib in an interrupted or continuous approach prolonged the prognosis. ${ }^{22,36-40}$ However, the result of the sequential approach for sorafenib in combination with TACE is controversial. Due to delays in starting sorafenib

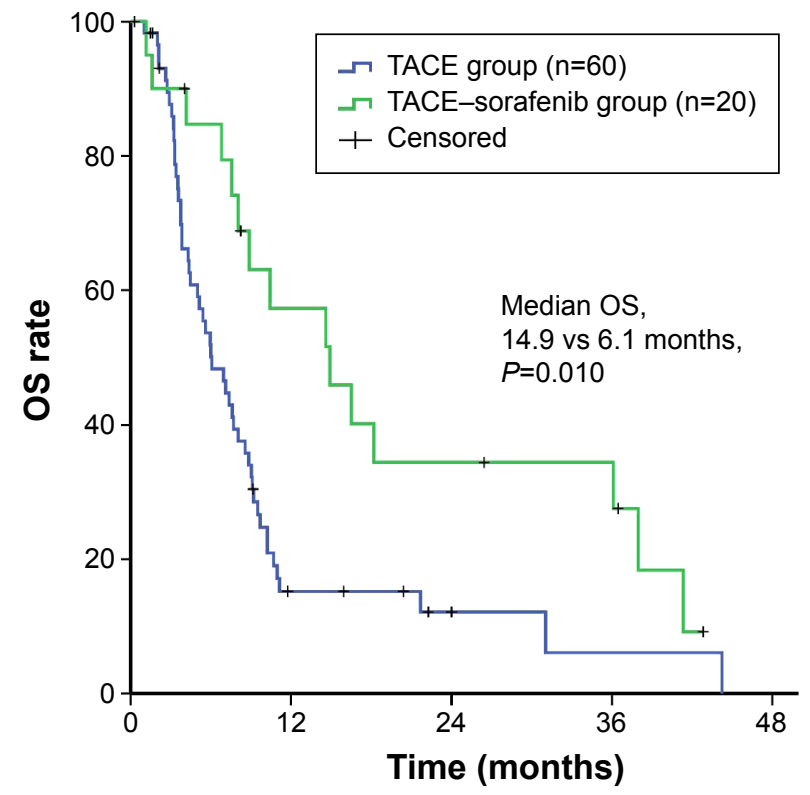

Figure I Kaplan-Meier curves of OS for patients with HCC and HVTT in the TACE-sorafenib group or the TACE group.

Notes: TACE-sorafenib group: median OS =14.9 months; TACE group: median OS $=6.1$ months. $P=0.010$.

Abbreviations: OS, overall survival; HCC, hepatocellular carcinoma; HVTT, hepatic vein tumor thrombus; TACE, transarterial chemoembolization. 
Table 4 Treatment outcomes for patients with HVTT

\begin{tabular}{|c|c|c|c|}
\hline Variables & TACE-sorafenib group $(n=20)$ & TACE group $(n=60)$ & $P$-value \\
\hline Best tumor response, $n(\%)$ & & & 0.014 \\
\hline$C R$ & $0(0)$ & 1 & \\
\hline PR & $10(50)$ & 11 & \\
\hline SD & $6(30)$ & 14 & \\
\hline $\mathrm{DCR}(\mathrm{CR}+\mathrm{PR}+\mathrm{SD})$ & $16(80)$ & $26(43.3)$ & 0.004 \\
\hline Median TTP $(95 \% \mathrm{Cl})$ (months) & $4.9(3.7-6.0)$ & $2.4(1.3-3.4)$ & 0.016 \\
\hline
\end{tabular}

Abbreviations: HVTT, hepatic vein tumor thrombus; TACE, transarterial chemoembolization; CR, complete response; PR, partial response; SD, stable disease; DCR, disease control rate; TTP, time to progression; $\mathrm{Cl}$, confidence interval.

( $>9$ weeks) after TACE, Kudo et al showed that sorafenib did not significantly prolong survival in patients who responded well to TACE. ${ }^{41}$ However, Sansonno et al indicated that TACE followed by sorafenib treatment resulted in a longer TTP in patients with intermediate-stage hepatitis $\mathrm{C}$ virus-related HCC. $^{42}$ Based on the two approaches for combination with TACE, our study showed that TACE combined with sorafenib could improve the outcomes of patients with advanced HCC and HVTT. Our study also showed that sorafenib as an adjuvant therapy following TACE keeps promise as a useful strategy for the treatment of patients with HCC and HVTT.

One aspect of the study that should be considered is that the patients were still treated with sorafenib, despite disease progression, until deterioration was observed in the patient Child-Pugh score to C or the ECOG PS score to 4, or until the occurrence of intolerable AEs or death. ${ }^{13}$ With informed

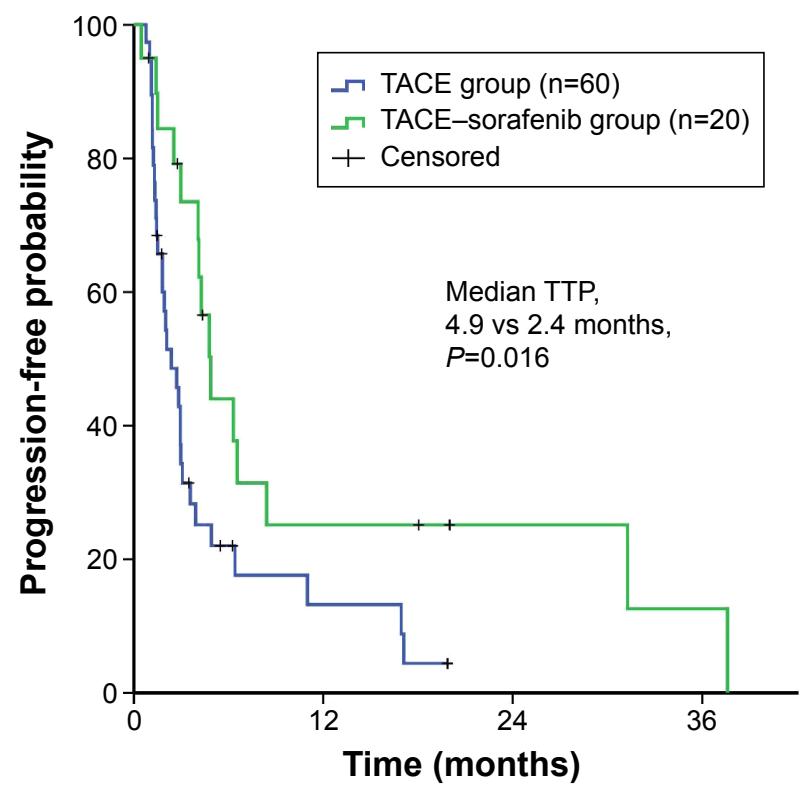

Figure 2 Kaplan-Meier curves of TTP for patients with HCC and HVTT in the TACE-sorafenib group or the TACE group.

Notes: TACE-sorafenib group: median TTP $=4.9$ months. TACE group: median TTP $=2.4$ months. $P=0.016$.

Abbreviations: TTP, time to progression; HCC, hepatocellular carcinoma; HVTT, hepatic vein tumor thrombus; TACE, transarterial chemoembolization. consent, sorafenib was continued in the study following PD, as no second-line therapy was established for HCC patients in the event of sorafenib failure. Several studies confirmed the feasibility of this continual treatment strategy. ${ }^{13,26,43}$ However, it is unknown whether the benefit of sorafenib remains after disease progression. Thus, further study is required.

In terms of safety, our study showed that sorafenib administration following TACE was well tolerated generally and led to only manageable side effects in patients with HCC and HVTT, provided that dose adjustment was permitted. The most common drug-related AEs noticed were HFSR, diarrhea, fatigue, and alopecia; these were similar to those reported in previous studies with sorafenib as the monotherapy $y^{3,444,45}$ and with sorafenib administered in combination with TACE. ${ }^{22,36,39}$ These AEs were predominantly of grade 1 or 2 , and grade 3 or 4 AEs were manageable with dose adjustment or interruption of sorafenib administration. Furthermore, TACE-sorafenib did not increase the TACErelated AEs in patients with HCC and HVTT.

However, there were some limitations to the current study. It is a retrospective and not a prospective study, and the choice of treatments was determined by the patients. The study was performed with case-matched controls to minimize potential bias. Still, certain deviations might have existed in our results. Moreover, the sample size of our single-center trial may not be large enough, although it was associated with multiple variables. In addition, maybe due to the relatively small sample size, our study failed to reveal the location of HVTT as a significant predictor of the OS. Therefore, a prospective trial with a larger sample size is required in future.

In conclusion, this study aimed to minimize bias by basing the analysis on case-matched controls. The results indicate that addition of sorafenib to TACE therapy significantly improves TTP and OS in patients with advanced HCC and HVTT. There were no unexpected AEs and no drug-related deaths, and all safety-related events were manageable. We suggest that a prospective clinical trial with a larger sample 
Table 5 Univariate and multivariate analysis of risk factors for overall survival

\begin{tabular}{|c|c|c|c|c|}
\hline \multirow[t]{3}{*}{ Variables } & \multirow{2}{*}{$\begin{array}{l}\text { Univariate analysis } \\
P \text {-value } \\
\end{array}$} & \multicolumn{3}{|c|}{ Multivariate analysis } \\
\hline & & HR & $95 \% \mathrm{Cl}$ & $P$-value \\
\hline & Log-rank & & & Cox regression \\
\hline Age (years) $(\leq 50 />50)$ & 0.161 & & & \\
\hline Sex (male/female) & 0.856 & & & \\
\hline ECOG performance $(0 / I)$ & 0.305 & & & \\
\hline Hepatitis B carrier (yes/no) & 0.630 & & & \\
\hline Liver cirrhosis (yes/no) & 0.309 & & & \\
\hline Platelet count $(\leq 100 />100)\left(10^{9} / \mathrm{L}\right)$ & 0.456 & & & \\
\hline Prothrombin time ( $\leq />14)$ (seconds) & 0.306 & & & \\
\hline Alanine aminotransferase $(\leq 40 />40)(\mathrm{U} / \mathrm{L})$ & 0.096 & & & \\
\hline Serum albumin $(\leq 35 />35)(g / L)$ & 0.512 & & & \\
\hline Total bilirubin $(\leq 20 />20)(\mathrm{mmol} / \mathrm{L})$ & 0.417 & & & \\
\hline Alpha-fetoprotein level $(\leq 400 />400)(\mathrm{ng} / \mathrm{mL})$ & 0.250 & & & \\
\hline Tumor size $(\leq 10 />10)(\mathrm{cm})$ & 0.745 & & & \\
\hline Tumor number $(\leq \mid />1)$ & 0.645 & & & \\
\hline Tumor extent (unilobar/bilobar) & 0.847 & & & \\
\hline Combined PVTT (yes/no) & 0.034 & & & \\
\hline Location of HVTT (Vv2/Vv3) & 0.494 & & & \\
\hline Extrahepatic metastasis (yes/no) & 0.206 & & & \\
\hline Treatment allocation (TACE/TACE-sorafenib) & 0.010 & 2.16 & $1.18-3.94$ & 0.012 \\
\hline
\end{tabular}

Notes: Vv2: the first branch of the hepatic vein branching from the inferior vena cava, including the right, left, and middle hepatic vein, the inferior right hepatic vein, or the short hepatic veins. Vv3: the inferior vena cava.

Abbreviations: HR, hazard ratio; Cl, confidence interval; ECOG, Eastern Cooperative Oncology Group; PVTT, portal vein tumor thrombus; HVTT, hepatic vein tumor thrombus; TACE, transarterial chemoembolization.

size would be required to further verify these results and the optimal sequence of such combination.

\section{Acknowledgments}

This study was supported by grants from the National Natural Science Foundation of China (No 81172037/H1606, No 81272639, and No 81572385), Guangdong Province Science and Technology Project of China (No 2013B021800159), and the Fundamental Research Funds for the Central Universities of China (No 13ykzd24).

\section{Disclosure}

The authors report no conflicts of interest in this work.

\section{References}

1. Jemal A, Bray F, Center MM, Ferlay J, Ward E, Forman D. Global cancer statistics. CA Cancer J Clin. 2011;61(2):69-90.

2. Bruix J, Sherman M; Practice Guidelines Committee, American Association for the study of Liver Diseases. Management of hepatocellular carcinoma. Hepatology. 2005;42(5):1208-1236.

3. Cheng AL, Kang YK, Chen Z, et al. Efficacy and safety of sorafenib in patients in the Asia-Pacific region with advanced hepatocellular carcinoma: a phase III randomised, double-blind, placebo-controlled trial. Lancet Oncol. 2009;10(1):25-34.

4. Llovet JM, Ricci S, Mazzaferro V, et al. Sorafenib in advanced hepatocellular carcinoma. N Engl J Med. 2008;359(4):378-390.

5. Bruix J, Sherman M; American Association for the Study of Liver Diseases. Management of hepatocellular carcinoma: an update. Hepatology. 2011;53(3):1020-1022.
6. Lee HS, Kim JS, Choi IJ, Chung JW, Park JH, Kim CY. The safety and efficacy of transcatheter arterial chemoembolization in the treatment of patients with hepatocellular carcinoma and main portal vein obstruction. A prospective controlled study. Cancer. 1997;79(11):2087-2094.

7. Luo J, Guo RP, Lai ECH, et al. Transarterial chemoembolization for unresectable hepatocellular carcinoma with portal vein tumor thrombosis: a prospective comparative study. Ann Surg Oncol. 2011; 18(2):413-420.

8. Chung SM, Yoon CJ, Lee SS, et al. Treatment outcomes of transcatheter arterial chemoembolization for hepatocellular carcinoma that invades hepatic vein or inferior vena cava. Cardiovasc Intervent Radiol. 2014; 37(6):1507-1515.

9. Kim HC, Lee JH, Chung JW, et al. Transarterial chemoembolization with additional cisplatin infusion for hepatocellular carcinoma invading the hepatic vein. J Vasc Interv Radiol. 2013;24(2):274-283.

10. Chern MC, Chuang VP, Cheng T, Lin ZH, Lin YM. Transcatheter arterial chemoembolization for advanced hepatocellular carcinoma with inferior vena cava and right atrial tumors. Cardiovasc Intervent Radiol. 2008;31(4):735-744.

11. Carmeliet $P$, Jain RK. Molecular mechanisms and clinical applications of angiogenesis. Nature. 2011;473(7347):298-307.

12. Shim JH, Park JW, Kim JH, et al. Association between increment of serum VEGF level and prognosis after transcatheter arterial chemoembolization in hepatocellular carcinoma patients. Cancer Sci. 2008; 99(10):2037-2044.

13. Shao W, Zhang F, Cong N, Li J, Song J. Transarterial chemoembolization combined with sorafenib for advanced hepatocellular carcinoma. Oncol Lett. 2014;8(5):2263-2266.

14. Guo JH, Zhu X, Li XT, Yang RJ. Impact of serum vascular endothelial growth factor on prognosis in patients with unresectable hepatocellular carcinoma after transarterial chemoembolization. Chin J Cancer Res. 2012;24(1):36-43.

15. Pan T, Li XS, Xie QK, et al. Safety and efficacy of transarterial chemoembolization plus sorafenib for hepatocellular carcinoma with portal venous tumour thrombus. Clin Radiol. 2014;69(12):e553-e561. 
16. Dufour JF, Hoppe H, Heim MH, et al. Continuous administration of sorafenib in combination with transarterial chemoembolization in patients with hepatocellular carcinoma: results of a phase I study. Oncologist. 2010;15(11):1198-1204.

17. McCubrey JA, Steelman LS, Abrams SL, et al. Roles of the RAF/MEK/ ERK and PI3K/PTEN/AKT pathways in malignant transformation and drug resistance. Adv Enzyme Regul. 2006;46:249-279.

18. Schmid I, Haberle B, Albert MH, et al. Sorafenib and cisplatin/doxorubicin (PLADO) in pediatric hepatocellular carcinoma. Pediatr Blood Cancer. 2012;58(4):539-544.

19. Sun W, Powell M, O’Dwyer PJ, Catalano P, Ansari RH, Benson AB 3rd. Phase II study of sorafenib in combination with docetaxel and cisplatin in the treatment of metastatic or advanced gastric and gastroesophageal junction adenocarcinoma: ECOG 5203. J Clin Oncol. 2010; 28(18):2947-2951.

20. Paz-Ares LG, Biesma B, Heigener D, et al. Phase III, randomized, double-blind, placebo-controlled trial of gemcitabine/cisplatin alone or with sorafenib for the first-line treatment of advanced, nonsquamous non-small-cell lung cancer. J Clin Oncol. 2012;30(25):3084-3092.

21. Xue C, Huang Y, Huang PY, et al. Phase II study of sorafenib in combination with cisplatin and 5-fluorouracil to treat recurrent or metastatic nasopharyngeal carcinoma. Ann Oncol. 2013;24(4):1055-1061.

22. Zhu K, Chen J, Lai L, et al. Hepatocellular carcinoma with portal vein tumor thrombus: treatment with transarterial chemoembolization combined with sorafenib - a retrospective controlled study. Radiology. 2014;272(1):284-293.

23. Liver Cancer Study Group of Japan. Primary liver cancer in Japan. Clinicopathologic features and results of surgical treatment. Ann Surg. 1990;211(3):277-287.

24. Roayaie S, Jibara G, Taouli B, Schwartz M. Resection of hepatocellular carcinoma with macroscopic vascular invasion. Ann Surg Oncol. 2013;20(12):3754-3760.

25. Kokudo T, Hasegawa K, Yamamoto S, et al. Surgical treatment of hepatocellular carcinoma associated with hepatic vein tumor thrombosis. J Hepatol. 2014;61(3):583-588

26. Gao HJ, Xu L, Zhang YJ, Chen MS. Long-term survival of patients with hepatocellular carcinoma with inferior vena cava tumor thrombus treated with sorafenib combined with transarterial chemoembolization: report of two cases and literature review. Chin J Cancer. 2014;33(5):259-264.

27. Kudo M, Izumi N, Kokudo N, et al. Management of hepatocellular carcinoma in Japan: Consensus-Based Clinical Practice Guidelines proposed by the Japan Society of Hepatology (JSH) 2010 updated version. Dig Dis. 2011;29(3):339-364.

28. Lencioni R, Llovet JM. Modified RECIST (mRECIST) assessment for hepatocellular carcinoma. Semin Liver Dis. 2010;30(1):52-60.

29. Institute NC. Common terminology criteria for adverse events v3.0 (CTCAE). Cancer. 2006;0:0-71.

30. Abou-Alfa GK. TACE and sorafenib: a good marriage? J Clin Oncol. 2011;29(30):3949-3952.

31. Choi GH, Shim JH, Kim MJ, et al. Sorafenib alone versus sorafenib combined with transarterial chemoembolization for advanced-stage hepatocellular carcinoma: results of propensity score analyses. Radiology. 2013;269(2):603-611.
32. Wang B, Xu H, Gao ZQ, Ning HF, Sun YQ, Cao GW. Increased expression of vascular endothelial growth factor in hepatocellular carcinoma after transcatheter arterial chemoembolization. Acta Radiol. 2008;49(5):523-529.

33. Virmani S, Rhee TK, Ryu RK, et al. Comparison of hypoxia-inducible factor-1alpha expression before and after transcatheter arterial embolization in rabbit VX2 liver tumors. $J$ Vasc Interv Radiol. 2008; 19(10):1483-1489.

34. Fernandez M, Semela D, Bruix J, Colle I, Pinzani M, Bosch J. Angiogenesis in liver disease. J Hepatol. 2009;50(3):604-620.

35. Strebel BM, Dufour JF. Combined approach to hepatocellular carcinoma: a new treatment concept for nonresectable disease. Expert Rev Anticancer Ther. 2008;8(11):1743-1749.

36. Cabrera R, Pannu DS, Caridi J, et al. The combination of sorafenib with transarterial chemoembolisation for hepatocellular carcinoma. Aliment Pharmacol Ther. 2011;34(2):205-213.

37. Qu XD, Chen CS, Wang JH, et al. The efficacy of TACE combined sorafenib in advanced stages hepatocellullar carcinoma. BMC Cancer. 2012;12:263.

38. Chao Y, Chung YH, Han G, et al. The combination of transcatheter arterial chemoembolization and sorafenib is well tolerated and effective in Asian patients with hepatocellular carcinoma: final results of the START trial. Int J Cancer. 2015;136(6):1458-1467.

39. Zhao Y, Wang WJ, Guan S, et al. Sorafenib combined with transarterial chemoembolization for the treatment of advanced hepatocellular carcinoma: a large-scale multicenter study of 222 patients. Ann Oncol. 2013;24(7):1786-1792.

40. Bai W, Wang YJ, Zhao Y, et al. Sorafenib in combination with transarterial chemoembolization improves the survival of patients with unresectable hepatocellular carcinoma: a propensity score matching study. J Dig Dis. 2013;14(4):181-190.

41. Kudo M, Imanaka K, Chida N, et al. Phase III study of sorafenib after transarterial chemoembolisation in Japanese and Korean patients with unresectable hepatocellular carcinoma. Eur J Cancer. 2011;47(14):2117-2127.

42. Sansonno D, Lauletta G, Russi S, Conteduca V, Sansonno L, Dammacco F. Transarterial chemoembolization plus sorafenib: a sequential therapeutic scheme for HCV-related intermediate-stage hepatocellular carcinoma: a randomized clinical trial. Oncologist. 2012; 17(3):359-366.

43. Welker MW, Zangos S, Kriener S, Monch C, Zeuzem S, Trojan J. Sequential therapy of transarterial chemoembolisation and sorafenib in intermediate stage hepatocellular carcinoma. J Gastrointest Cancer. 2010;41(2):149-152.

44. Kee KM, Hung CH, Wang JH, Lu SN. Serial changes of clinical parameters in a patient with advanced hepatocellular carcinoma with portal vein thrombosis achieving complete response after treatment with sorafenib. Onco Targets Ther. 2014;7:829-834.

45. Almhanna K, Philip PA. Safety and efficacy of sorafenib in the treatment of hepatocellular carcinoma. Onco Targets Ther. 2009;2:261-267.

\section{Dovepress}

\section{Publish your work in this journal}

OncoTargets and Therapy is an international, peer-reviewed, open access journal focusing on the pathological basis of all cancers, potential targets for therapy and treatment protocols employed to improve the management of cancer patients. The journal also focuses on the impact of management programs and new therapeutic agents and protocols on

Submit your manuscript here: http://www.dovepress.com/oncotargets-and-therapy-journal patient perspectives such as quality of life, adherence and satisfaction The manuscript management system is completely online and includes a very quick and fair peer-review system, which is all easy to use. Visit http://www.dovepress.com/testimonials.php to read real quotes from published authors. 